Nonlinear Processes in Geophysics, 12, 947-953, 2005

SRef-ID: $1607-7946 / \mathrm{npg} / 2005-12-947$

European Geosciences Union

(c) 2005 Author(s). This work is licensed

under a Creative Commons License.

\title{
Rossby-wave turbulence in a rapidly rotating sphere
}

\author{
N. Schaeffer and P. Cardin \\ LGIT, Observatoire de Grenoble, Université Joseph Fourier, Grenoble, France
}

Received: 1 August 2005 - Revised: 19 October 2005 - Accepted: 19 October 2005 - Published: 4 November 2005

Part of Special Issue "Turbulent transport in geosciences"

\begin{abstract}
We use a quasi-geostrophic numerical model to study the turbulence of rotating flows in a sphere, with realistic Ekman friction and bulk viscous dissipation. The forcing is caused by the destabilization of an axisymmetric Stewartson shear layer, generated by differential rotation, resulting in a forcing at rather large scales.

The equilibrium regime is strongly anisotropic and inhomogeneous but exhibits a steep $m^{-5}$ spectrum in the azimuthal (periodic) direction, at scales smaller than the injection scale. This spectrum has been proposed by Rhines for a Rossby wave turbulence. For some parameter range, we observe a turbulent flow dominated by a large scale vortex located in the shear layer, reminding us of the Great Red Spot of Jupiter.
\end{abstract}

\section{Introduction}

In many geophysical flows, global rotation deeply modifies the nature of turbulence. This is the case for planetary flows at large scales, such as the Earth atmosphere, ocean or fluid metallic core, but also the giant gaseous planets like Jupiter or Saturn.

Because of the dominance of the Coriolis force (i.e. $R o=U / L \Omega \ll 1, E k=v / L^{2} \Omega \ll 1$, where $U$ is a typical velocity of the flow, $L$ a typical length scale, $\Omega$ the rotation rate of the system, and $v$ the kinematic viscosity of the fluid), flow variations along the rotation axis are inhibited (TaylorProudman constraint) resulting in a nearly two-dimensional turbulence (Greenspan, 1968). An inverse energy cascade takes place, so that large scale structures build up with almost no bulk dissipation. In addition, when the Coriolis parameter is not constant in the whole domain (owing to the presence of a $\beta$-effect due to either a varying angle between the rotation vector and the thin layer or a height variation as in Sect. 2), the incompressible flow obeys a partic-

Correspondence to: P. Cardin

(philippe.cardin@ujf-grenoble.fr) ular wave motion, the so-called Rossby waves (Greenspan, 1968). Actually, the $\beta$-effect adds a vortex-stretching term in the 2D Navier-Stokes equation, leading to a different dynamics (Schaeffer and Cardin, 2005). Rhines (1975) proposed that the inverse cascade is blocked by the $\beta$-effect at the Rhines wave number $k_{\beta} \equiv \sqrt{\beta / 2 U}$, and he suggested that turbulence in equilibrium with Rossby waves may exhibit an energy spectrum $E(k) \sim 4 \beta^{2} k^{-5}$ (where $k$ is the wave number). One must consider two types of simplified models of rotating flows in which Rossby waves may exist.

On the one hand, the shallow water model has been developped to model the large scale flows in the Earth atmosphere or oceans (where the $\beta$-effect comes from the variation of the projection of the rotation vector normal to the layer). This model has already been used to study turbulence using small scale forcing: Huang et al. (2001) report $n^{-5}$ spectra in numerical simulations of two-dimensional turbulence on the surface of a rotating sphere (where $n$ is the order of the spherical harmonic). Even though the spectrum shows the same exponent, this is not believed to be Rhines spectrum (Galperin et al., 2001).

On the other hand, the quasi-geostrophic (QG) approximation has been used to study deep flows in a rotating frame, like the liquid metal motion in the Earth's Core or the atmosphere dynamics of giant planets (where the $\beta$-effect comes from depth variations of the fluid). This kind of model has been used with small variations by many authors, to study thermal convection of rapidly rotating spheres (Busse, 1970; Aubert et al., 2003), to investigate the instabilities of shear layers (Busse, 1968; Schaeffer and Cardin, 2005), to model oceanic flows over topography (Kiss, 2003) or to reproduce the zonal jets of Jupiter (Yano et al., 2003).

These two kinds of models share the same governing equation, but have different boundary conditions. As a result, QG models and thin shell models lead to flows which have many features in common but which are not identical. This paper focuses on deep rotating turbulent flows filling a sphere, using a QG-model and large scale forcing. Recently, in (deep) rotating turbulence experiments (Baroud et al., 2002, 2003), 


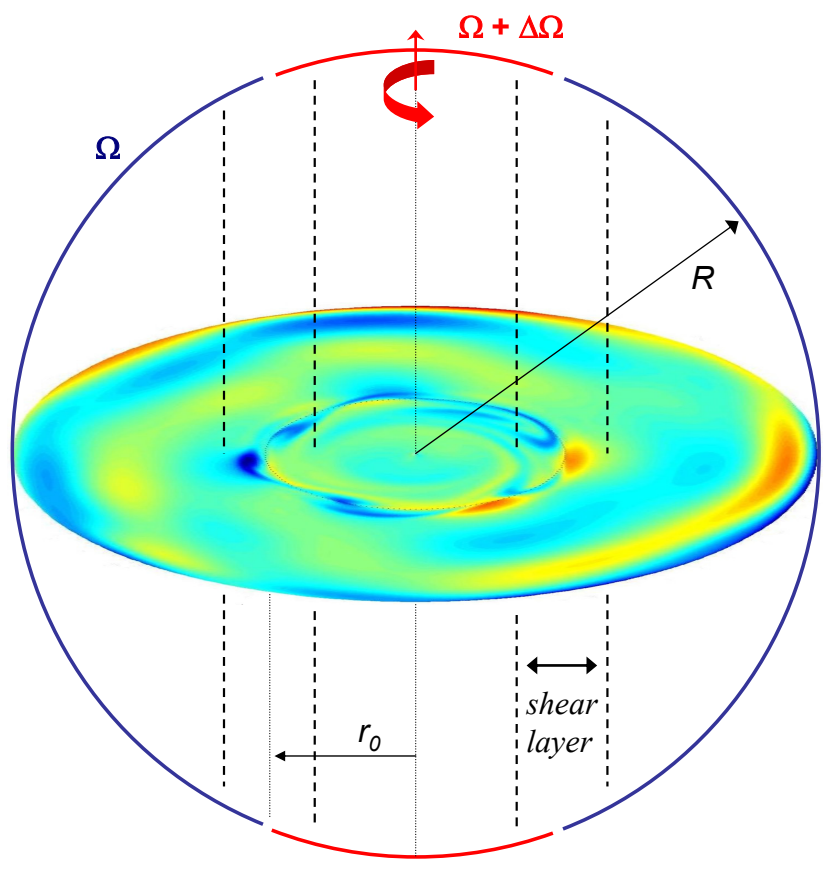

Fig. 1. Schematic setup with an equatorial vorticity map obtained from the numerical calculations.

it has been shown that for Rossby number up to 0.1 , the velocity fluctuations recorded by hot-film probes are strongly correlated along the rotation axis direction, suggesting that a QG-model may describe such turbulent flows quite well.

Usually the QG approximation is restricted to asymptotically small slopes, but by averaging carefully the mass conservation equation and the Navier-Stokes equation, it can be extended to slopes up to order one. In addition to the bulk viscous force, we derive the Ekman friction term from the Greenspan (1968) formula, providing a quite realistic dissipation at all scales. This improved QG-model is described in details in Sect. 2.

We apply our QG-model to a fluid-filled sphere rotating at an angular velocity $\Omega$, while the polar caps rotate slightly faster or slower (by an amount $\Delta \Omega$ ) than the equatorial parts (Fig. 1). The differential rotation is controlled by the Rossby number $R o=\Delta \Omega / \Omega$, while the importance of the viscous forces compared to the Coriolis force is given by the Ekman number $E k=v / R^{2} \Omega$.

In a previous paper (Schaeffer and Cardin, 2005), we studied the instabilities of the Stewartson layer, summarized in Sect. 3. Here we increase the forcing (i.e. Ro) to study the turbulence regime in such systems (Sect. 4), keeping in mind that zonal movements and Ekman friction influence the shape of the spectrum (Danilov and Gurarie, 2004).

\section{The quasi-geostrophic model}

We want to describe the fluid dynamics of a rotating fluid in a spherical container. We use the cylindrical coordinate system $\left(\boldsymbol{e}_{\boldsymbol{r}}, \boldsymbol{e}_{\boldsymbol{\phi}}, \boldsymbol{e}_{z}\right)$, with $\boldsymbol{e}_{z}$ parallel to $\boldsymbol{\Omega}$. The half height of
Table 1. Most extreme computations for different Ekman numbers. $N_{R}$ and $m_{\text {max }}$ are the radial (physical) and azimuthal (spectral) resolution of the computation performed with time-step $d t$ (in rotation periods units) at Rossby number Ro. The flow is then dominated by wave number $m_{d}$. The Reynolds number $R e_{r m s}$ is computed from non-zonal rms velocities. For comparison purposes the critical Rossby number $R o_{c}$ as well as the azimuthal wave number at the onset $m_{c}$ are given.

\begin{tabular}{lccccccccc}
\hline$i d$ & $E k$ & $N_{R}$ & $m_{\max }$ & $d t$ & $R o$ & $R o_{c}$ & $R e_{r m s}$ & $m_{d}$ & $m_{c}$ \\
\hline$e 6^{+}$ & $10^{-6}$ & 400 & 128 & 0.1 & 0.04 & 0.008 & $1.8510^{3}$ & 1 & 10 \\
$e 6^{-}$ & $10^{-6}$ & 400 & 64 & 0.1 & -0.08 & -0.01 & $1.8510^{3}$ & 3 & 8 \\
$e 7^{+}$ & $10^{-7}$ & 500 & 170 & 0.05 & 0.015 & 0.002 & $710^{3}$ & 2 & 19 \\
$e 8^{+}$ & $10^{-8}$ & 600 & 170 & 0.01 & 0.02 & $610^{-4}$ & $6010^{3}$ & 2 & 34 \\
$e 8^{-}$ & $10^{-8}$ & 600 & 170 & 0.05 & -0.02 & $-710^{-4}$ & $4010^{3}$ & 5 & 31 \\
\hline
\end{tabular}

the spherical container is then $L(r)=\sqrt{1-r^{2}}$. The quasigeostrophic model we use is derived by carefully averaging along the rotation axis both the incompressible NavierStokes equation and the mass conservation equation (Schaeffer, 2004; Schaeffer and Cardin, 2005). With $\boldsymbol{u}$ the velocity field and $\omega$ the vorticity along the rotation axis $\left(\boldsymbol{\omega} . \boldsymbol{e}_{z}\right)$, and under the assumption that $\boldsymbol{u} \times \boldsymbol{e}_{z}$ is independent of $z$, we obtain

$$
\begin{aligned}
\frac{\partial \omega}{\partial t}+\left(u_{r} \frac{\partial \omega}{\partial r}+\frac{u_{\phi}}{r} \frac{\partial \omega}{\partial \phi}\right)- & \\
(2+\omega)\left\langle\frac{\partial u_{z}}{\partial z}\right\rangle & =E k \nabla^{2} \omega
\end{aligned}
$$

with $\langle$.$\rangle the z$-averaging operator and

$$
\begin{aligned}
u_{r} & \equiv \frac{1}{r} \frac{\partial \psi}{\partial \phi} \\
u_{\phi} & =-\frac{\partial \psi}{\partial r}-\beta \psi \\
\omega & =-\nabla^{2} \psi-\beta\left(\frac{\partial \psi}{\partial r}+\frac{\psi}{r}\right)-\frac{d \beta}{d r} \psi
\end{aligned}
$$

where $\psi$ is a pseudo-stream function and $\beta$ is related to the slope of the container by

$\left.\beta \equiv \frac{1}{L} \frac{d L}{d r}\right|_{z=L}=\frac{-r}{1-r^{2}}$

Using the Ekman pumping boundary condition given by Greenspan (1968), the vortex stretching term is also independent of $z$ and reads:

$$
\begin{aligned}
\frac{\partial u_{z}}{\partial z}= & \beta u_{r}-\frac{E k^{1 / 2}}{2\left(1-r^{2}\right)^{3 / 4}} \\
& \left(\omega+\beta\left(\frac{\partial u_{r}}{\partial \phi}-\frac{1}{2} u_{\phi}\right)+\frac{5 r}{2\left(1-r^{2}\right)^{3 / 2}} u_{r}\right)
\end{aligned}
$$

This model is then implemented using a Fourier expansion $\left(m_{\max }\right.$ modes) in the azimuthal, periodic direction and a finite difference scheme ( $N_{R}$ grid points) in the radial direction. See Table 1 for typical values of $N_{R}$ and $m_{\max }$. Note that 


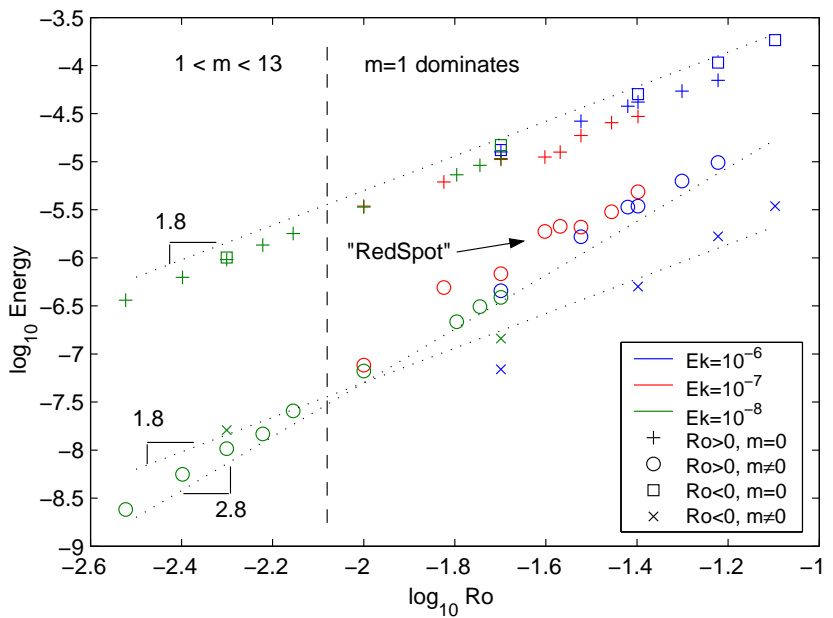

Fig. 2. Total kinetic energy vs. Rossby number for different flows far from the instability threshold. RedSpot stands for the very stationary regime.

neither hyper- nor hypo-viscosities are used, only bulk volume viscosity and realistic Ekman friction are implemented.

It can be shown that this model is self-consistent as long as $|\beta|$ is not too large compared to 1 . This is not true in the case of the sphere studied here, leading to a strong amplification of the vorticity near the equator (see Fig. 4). To avoid that problem, Yano et al. (2003) truncated the sphere before the equator, but we checked that with or without such artifact, the flow and the dynamics stayed nearly the same.

\section{Overview over results}

The sphere is split at cylindrical radius $r_{0}=0.35$, and while the outer part $\left(r>r_{0}\right)$ is at rest in the rotating frame, the inner part is rotating at an angular velocity $\Delta \Omega$. This differential rotation produces an axisymmetric shear layer located near $r_{0}$. This shear layer has been studied by Stewartson (1966) and is independent of $z$ at leading order (Fig. 1). At very small Rossby numbers, the Stewartson shear layer of width $\Delta \sim E^{1 / 4}$ is stable.

For a small fixed Ekman number $E k$, we increase the Rossby number $R o$. The parameter range studied here spans from $E k=10^{-6}$ to $E k=10^{-8}$, with $|R o|$ varying from the onset of instability $R o_{c}$ up to values close to 0.1 . This corresponds to $R o / R o_{c}$ up to about 30 and leads to rms values of the Reynolds number up to $610^{4}$. We will focus on five turbulent runs, which are presented in Table 1, but let us start with a brief overview over the behavior of the flow for increasing $R o$.

At $R o_{c} \sim \beta\left(r_{0}\right) E k^{1 / 2}$, the flow becomes unstable, and the instability is a single Rossby wave, with a well defined azimuthal wave-number $m \sim E k^{-1 / 4}$. Table 1 gives the values of the critical Rossby number $R o_{c}$ and azimuthal wave $m_{c}$ number at the onset of instability for different values of $E k$. It has been noticed that the radial structure depends on the sign of $R o$ : for $R o>0$ the disturbances fill the whole $r>r_{0}$

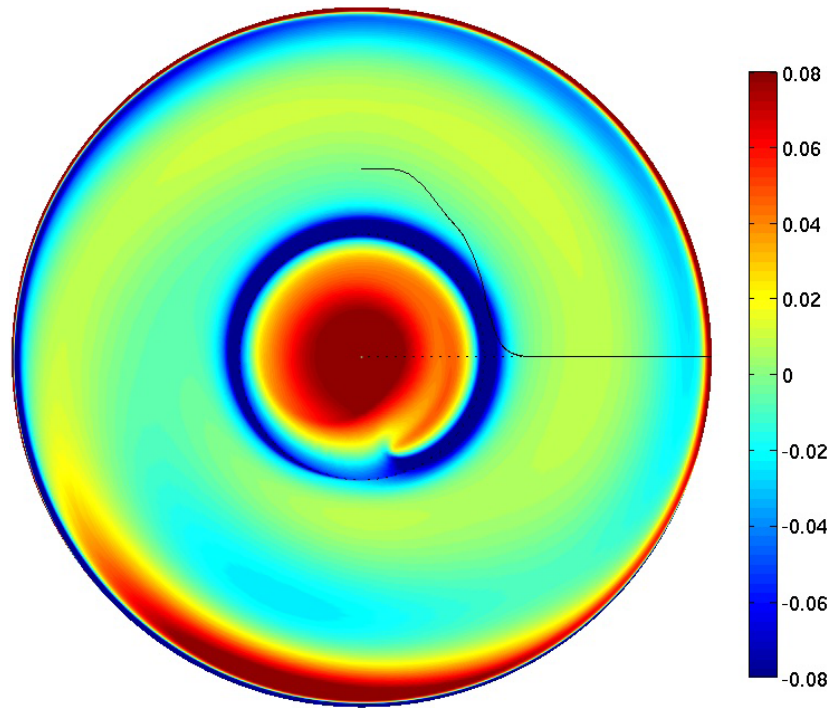

Fig. 3. Vorticity map (including $m=0$ ) for $E k=10^{-6}$, $R o=0.04$ ( $e 6^{+}$run, RedSpot regime). This run showed no evolution for one million rotation periods. The radial profile of the mean azimuthal velocity $\left(u_{\phi}, m=0\right)$ is given by the black line, and is normalized so that a radial size corresponds to $2 \mathrm{Ro}$.

area, whereas for $R o<0$ they stay more localized near $r=r_{0}$. A detailed study of the flow at the threshold can be found in Schaeffer and Cardin (2005).

Above the threshold, the dominant azimuthal wave number decreases when the Rossby number is increased. This behavior has also been observed by Hollerbach et al. (2004). Increasing the Rossby number further leads to periodic regimes, with a few excited modes. At even larger $R o$, the behavior becomes chaotic, with the dominant mode $m_{d}$ changing randomly, with large energy fluctuations, but still a quite narrow energy spectrum. Finally, for the strongest forcing compatible with the QG approach, one obtains a turbulent regime, that will be detailed in Sect. 4 .

The evolution of the total kinetic energy of the flow (integrated in the whole 3D domain) with various forcing is showed in Fig. 2. One may notice that regardless of the Ekman number, the energy of the zonal mode $(m=0)$ lies close to a $|R o|^{1.8}$ line. The non-zonal energy, however, seems to follow different power laws, depending on the sign of the Rossby number. $\left(|R o|^{1.8}\right.$ for $R o<0$ and $|R o|^{2.8}$ for $\left.R o>0\right)$.

\section{Rossby wave turbulence}

For very strong forcing, the energy spectrum becomes continuous, showing a significant inertial range. This is what we call a turbulent regime. The most extreme Rossby number for which we were able to compute the flow using our numerical model are given in Table 1, and all of these are turbulent, and the spectra were checked for good convergence.

The total energy of the flow may be highly time dependent. However, in the parameter space $(E k, R o)$ there are 

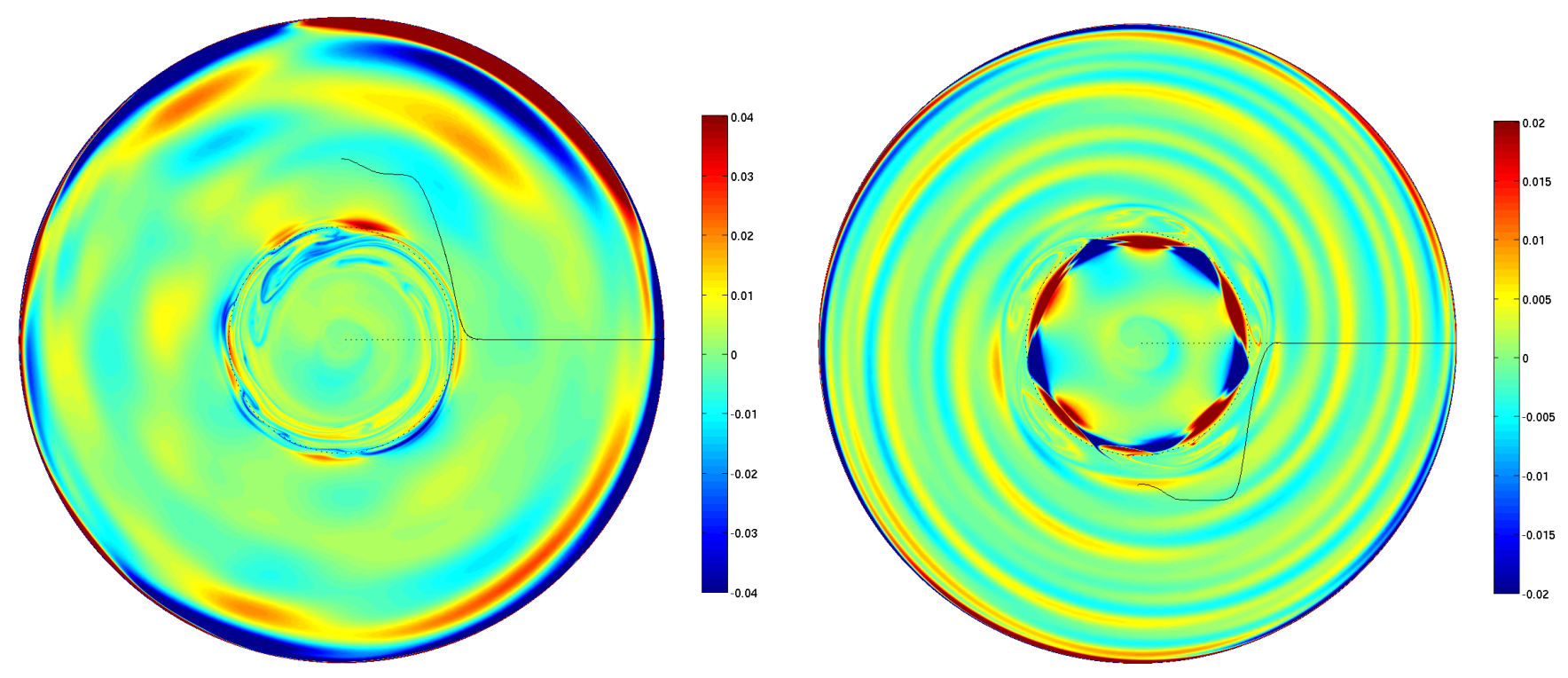

Fig. 4. Non-zonal vorticity maps (for $m \neq 0$ ) for $E=10^{-8}$ and $R o=0.02$ (left, $e 8^{+}$run) and $R o=-0.02$ (right, $e 8^{-}$run). The radial profile of the mean azimuthal velocity $\left(u_{\phi}, m=0\right)$ is given by the black line, and is normalized so that a radial size corresponds to $2 R o$.

islands of very stationary flows, whose only time evolution is an azimuthal drift. This kind of equilibrium reminds us of the Great Red Spot of Jupiter, that has been observed since the seventeenth century, that's why we call them RedSpot regimes (Fig. 2). Figure 3 shows one of these flows with a single big vortex, located near the shear. The corresponding run did not show any deviation after one million rotation periods. This kind of behavior has been obtained by a statistical approach in the case of 2D turbulence with neither $\beta$-effect nor dissipation (Sommeria et al., 1988; Robert and Sommeria, 1991).

\subsection{Vorticity maps}

Vorticity maps are represented in Figs. 3 and 4, they show the vorticity parallel to the rotation axis, in the equatorial plane. Let us recall that in our model this vorticity is invariant along the rotation axis, so that these maps contain all the information on a snapshot of the QG-flow.

These flows are dominated by large scale structures ( $m_{d}=1$ to $m_{d}=5$ ) but we observe the same strong asymmetry when changing the sign of $R o$, as the one at the onset of instability. Furthermore, the spiralization is also still present, and both asymmetry and spiralization were features of the Rossby wave nature of the instability (Schaeffer and Cardin, 2005).

The spiralization may be compared to the zonation in thinshell turbulence (Galperin et al., 2004) or deep convection (Read et al., 2004). Large scale flow features may not get larger than the Rhines scale in the direction of the $\beta$-effect, but are free to grow in the perpendicular direction. Indeed, the radial scale of the spiral arms is comparable to the Rhines scale $k_{\beta}$ given in Table 2 .

Very thin vortex filaments can be seen near the split radius $r_{0}$ (Fig. 4), sheared away from the large scale vortex by the strong zonal flow, and perhaps exciting Rossby waves at these small scales. The large eddies seem to be more stable and long-lasting for $R o<0$ than for $R o>0$, which is the usual observation: anticyclones are more stable than cyclones. Furthermore, regardless of the sign of $R o$, positive vorticity seems to gather at the center (near $r=0$ ), deforming the original differential rotation profile (see black lines in Figs. 3 and 4).

From these vorticity maps, one may question the homogeneity of this turbulence, as some structures seem to be linked with the shear layer. Figure 5 showing the evolution of the time-averaged velocity fluctuations with the radius, proves that the turbulent flow generated by this shear layer is far from homogeneous. In addition, the difference between azimuthal and radial velocity fluctuations shows an anisotropy of the turbulence. It is quite surprising that such time-averaged values still show strong radial dependence far from $r_{0}$, as in the $u_{\phi}$ fluctuations of $e 8^{+}$. This feature does not look like spiralization (which can be seen on a snapshot) but rather like zonation. However, why would some points exhibit more fluctuations than others?

\subsection{Spatial spectrum}

Some energy spectra from our QG-flow runs are represented in Fig. 6. These are instant spectra (not time-averaged), averaged over all radii, and showing a wide range where 
Table 2. Angular phase speed $c$ of the dominant mode $m_{d}$ in the turbulent runs (see Table 1), at a fixed $r$.

\begin{tabular}{lccccccc}
\hline id & $R o$ & $R o / R o c$ & $r$ & $c(r)$ & $\Omega(r)$ & $m_{d}$ & $k_{\beta}$ \\
\hline$e 6^{+}$ & 0.04 & 4.2 & 0.44 & 0.032 & $5.810^{-4}$ & 1 & 6 \\
$e 6^{-}$ & -0.08 & 7.3 & 0.44 & -0.013 & -0.0026 & 3 & 4 \\
$e 7^{+}$ & 0.015 & 6.5 & 0.40 & 0.012 & $3.610^{-5}$ & 2 & 9 \\
$e 8^{+}$ & 0.02 & 31.7 & 0.38 & 0.02 & 0.0014 & 2 & 8 \\
$e 8^{-}$ & -0.02 & 28.5 & 0.38 & -0.003 & -0.0037 & 5 & 7 \\
\hline
\end{tabular}

$E \sim C m^{-5}$. Moreover, it seems that all curves converge toward $C \sim 1.510^{-4}$ (within a factor 3 ).

It is also remarkable that the $e 6^{+}$spectrum, which lies in the RedSpot regime is very smooth compared to the other. When time averaged, all these spectrum become smooth (see Fig. 7).

Because our forcing is due to differential rotation, which is axisymmetric $(m=0)$, there is no small scale forcing. Hence, we can infer that the bump at large scales in the spectrum (Fig. 6) corresponds to the injection scale. To ensure this hypothesis, we computed the spectral energy transfer for our runs and it appeared that the small scales $\left(m>m_{d}\right)$ do drain energy from the large scale flow.

The $m^{-5}$ behavior of the spectra at scales smaller than the injection scale seems to be a very robust feature. On the other side of the injection scale, an inverse cascade transports energy towards the largest scales, where it is dissipated through Ekman friction. Note that the injection scale is very close to $k_{\beta}$, the blocking wave-number (Table 2), supporting the Rossby-wave $m^{-5}$ spectrum at smaller scales and an upward energy cascade at larger scales.

The viscous cutoff at small scales may be observed on the $e 6^{+}$spectrum (Fig. 6), but for performance issues the other runs were computed with a spectral resolution that makes the dissipative range invisible. One may also notice that there is no visible bump in the $e 6^{+}$spectrum, probably because the dominant scale is $m=1$.

\subsection{Rossby waves}

Many observed features suggest a Rossby wave turbulence. To perform another test, we computed the phase speed $c$ of the dominant Fourier mode $m_{d}$. This angular phase speed is computed in the rotating frame $(\Omega)$. Because of the strong zonal flow (the Stewartson layer), all the structures are advected, so that $c \neq 0$ does not mean that there is propagation. Actually, $c=0$ means that there is a wave propagating at a speed such that the advection is compensated. Furthermore, if $c$ does not depend on the radius, it means that the true phase speed depends on the radius in a way to compensate exactly the effect of advection. This is what is observed at the onset of instability, where the spiralization allows the phase speed to adjust to the local mean velocity, so that the structures are not torn apart.

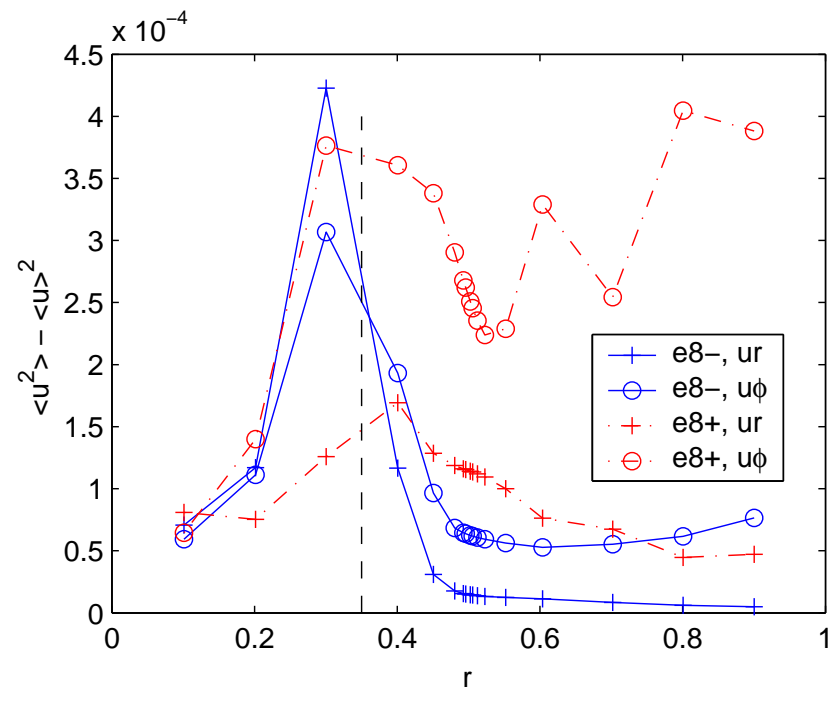

Fig. 5. Velocity fluctuations as a function of the radius, showing the strong inhomogeneity and anisotropy of the turbulence. The dotted line stands for the split radius at $r_{0}=0.35$.

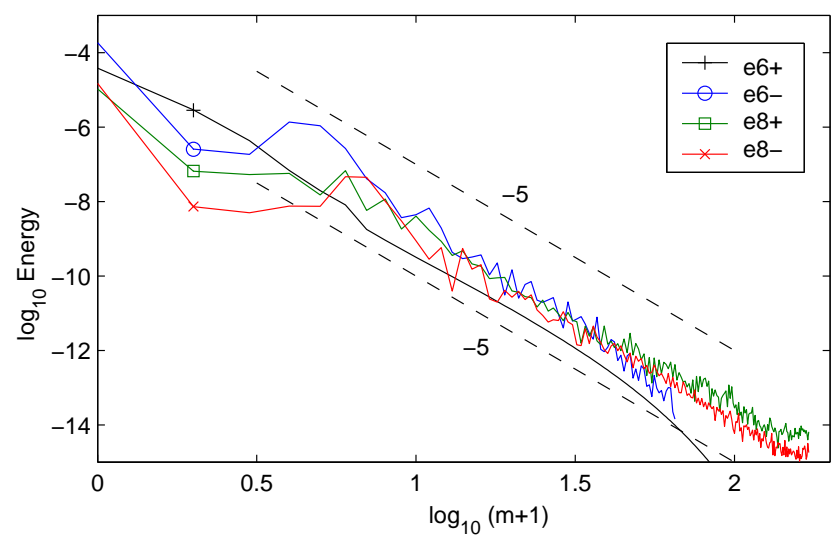

Fig. 6. Instant energy spectrum in the azimuthal direction averaged over radius $r$, for different parameters (see Table 1).

Phase speeds measured in our most turbulent runs, are given in Table 2. They are always lower than $R o$, but of the same order of magnitude. For $R o>0$, it is clear that phase speeds $c$ are greater than the local advection $\Omega(r)$. For $R o<0$ it is not that clear, but it seems (at least for the $e 6^{-}$case) that a prograde wave is propagating against the advection.

\section{Conclusions}

Thanks to our improved QG-model which includes the Ekman friction and the mass conservation, we are able to reach very turbulent flows $\left(R e_{r m s}>10^{4}\right)$ in our numerical runs at low $R o$ and very low $E k$, with neither hyper- nor hypoviscosities. For some parameters, very steady regimes are observed, reminiscent of the Jovian Great Red Spot, and extending previous theoretical results on rotating turbulence 


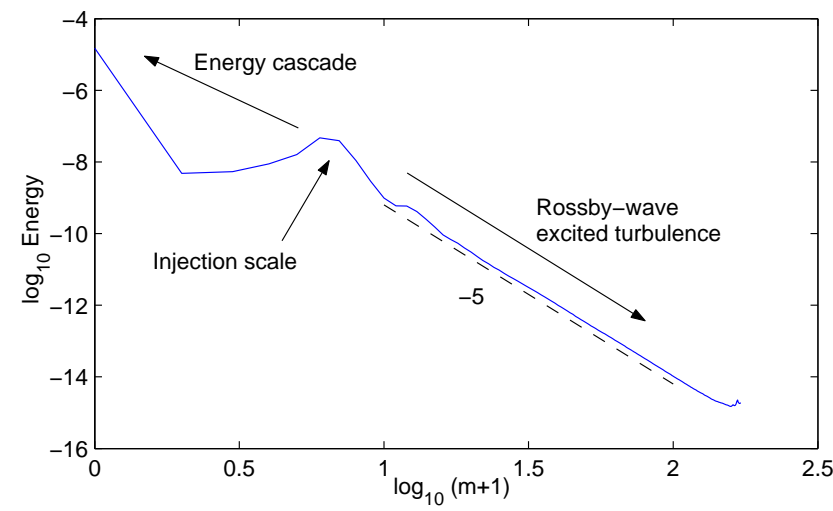

Fig. 7. Typical spectrum ( $e 8^{-}$run, space and time averaged) on top of which we added some phenomenology, to summarize the behavior of the observed turbulent flows.

(Robert and Sommeria, 1991), to Rossby-wave turbulence with Ekman friction.

The observed turbulence is strongly anisotropic and inhomogeneous, and the azimuthal energy spectrum seems to obey an $m^{-5}$ power law for wave numbers larger than the one corresponding to the injection scale, regardless of the sign of Ro. Once the axisymmetric flow is removed, we observe a wave-like behavior of the large scale features of the flow, with patterns drifting in the prograde azimuthal direction. This also seems to remain true for smaller scales. All the features observed seem to suggest a turbulence dominated by Rossby waves, as predicted by Rhines (1975), and for which very little other evidence has been found before.

An averaged spatial spectrum with some added phenomenology is presented in Fig. 7 showing the inertial range, and the injection scale. The strong rotation prevents any downward cascade to small scales where the energy could be dissipated. The energy then goes to large scale where it is dissipated by Ekman friction. Only a few Rossby waves (which may be excited in the way the primary instabilities are) are able to reach the smallest scales. In fact, the only movements allowed in such QG-model are geostrophic flows (zonal flows in our case, like the shear layer) and Rossby waves, so that it is no surprise to observe a turbulence dominated by these waves.

One remarkable feature is the difference between positive and negative differential rotation, which holds even far away from the instability threshold, suggesting that this kind of turbulence may keep memory of its generating mechanism.

In fluid turbulence, -5 spectrum seem to have at least two different origins: Rossby-wave, quasi-geostrophic turbulence (Rhines, 1975, and this paper) and quasi-onedimensional turbulence (Galperin et al., 2001; Huang et al., 2001). Even though both are described by the same equation, they are obviously of very different natures and hard to compare: the first kind of flow exhibits spiralization and an $m^{-5}$ spectrum in the direction perpendicular to the $\beta$-effect and at scales smaller than the injection scale, whereas the latter shows zonation and an $n^{-5}$ spectrum at scales between the injection scale and the Rhines blocking scale, in the opposite direction. Are these just two different regimes due to a different forcing scheme? Is it a geometrical effect? Is there a link between them? How does spiralization relates to zonation? Future studies should try to answer these open questions.

Acknowledgements. Calculations were performed at SCCI (Observatoire de Grenoble) and at IDRIS (CNRS). This work has been supported by the program "DyETI" of CNRS/INSU. We wish to thank H.-C. Nataf and P. Read for very useful comments.

Edited by: W.-G. Früh

Reviewed by: two referees

\section{References}

Aubert, J., Gillet, N., and Cardin, P.: Quasigeostrophic models of convection in rotating spherical shells, Geochem. Geophys. Geosyst., 4, 1052, doi:10.1029/2002GC000 456, 2003.

Baroud, C. N., Plapp, B. B., She, Z.-S., and Swinney, H. L.: Anomalous Self-Similarity in a Turbulent Rapidly Rotating Fluid, Phys. Rev. Lett., 88, 114 501, 2002.

Baroud, C. N., Plapp, B. B., She, Z.-S., and Swinney, H. L.: Scaling in three-dimensional and quasi-two-dimensional rotating turbulent flows, Phys. Fluids, 15, 2091-2104, 2003.

Busse, F. H.: Shear flow instabilities in rotating systems, J. Fluid Mech., 33, 577-589, 1968.

Busse, F. H.: Thermal instabilities in rapidly rotating systems, J. Fluid Mech., 44, 441-460, 1970.

Danilov, S. and Gurarie, D.: Scaling, spectra and zonal jets in betaplane turbulence, Phys. Fluids, 16, 2592-2603, 2004.

Galperin, B., Sukoriansky, S., and Huang, H.-P.: Universal $n^{-5}$ spectrum of zonal flows on giant planets, Phys. Fluids, 13, 15451548, 2001.

Galperin, B., Nakano, H., Huang, H.-P., and Sukoriansky, S.: The ubiquitous zonal jets in the atmospheres of giant planets and Earths oceans, Geophys. Res. Lett., 31, L13 303, doi:10.1029/2004GL019691, 2004.

Greenspan, H. P.: The theory of rotating fluids, Cambridge University Press, 43-51, 1968.

Hollerbach, R., Futterer, B., More, T., and Egbers, C.: Instabilities of the Stewartson layer Part 2. Supercritical mode transitions, Theor. Comput. Fluid Dyn., 18, 197-204, 2004.

Huang, H.-P., Galperin, B., and Sukoriansky, S.: Anisotropic spectra in two-dimensional turbulence on the surface of a rotating sphere, Phys. Fluids, 13, 225-240, 2001.

Kiss, A. E.: A modified quasigeostrophic formulation for weakly nonlinear barotropic flow with large-amplitude depth variations, Ocean Model., 5, 171-191, 2003.

Read, P. L., Yamazaki, Y. H., Lewis, S. R., Williams, P. D., Miki-Yamazaki, K., Sommeria, J., Didelle, H., and Fincham, A.: Jupiters and Saturns convectively driven banded jets in the laboratory, Geophys. Res. Lett., 31, L22 701, doi:10.1029/2004GL020 106, 2004.

Rhines, P. B.: Waves and turbulence on a beta-plane, J. Fluid Mech., 69, 417-443, 1975.

Robert, R. and Sommeria, J.: Statistical equilibrium states for 2dimensional flows, J. Fluid Mech., 229, 291-310, 1991.

Schaeffer, N.: Instabilités, turbulence et dynamo dans une couche de fluide cisaillée en rotation, Ph.D. thesis, Univer- 
sité Joseph Fourier, http://tel.ccsd.cnrs.fr/documents/archives0/ 00/01/06/84/, 2004.

Schaeffer, N. and Cardin, P.: Quasi-geostrophic model of the instabilities of the Stewartson layer in flat and depth varying containers, Phys. Fluids, 17, 104-111, 2005.

Sommeria, J., Meyers, S. D., and Swinney, H. L.: Laboratory simulation of Jupiter's Great Red Spot, Nature, 361, 689-693, 1988.
Stewartson, K.: On almost rigid rotations, Part 2, J. Fluid Mech., 26, 131-144, 1966.

Yano, J.-I., Talagrand, O., and Drossart, P.: Outer planets: Origins of atmospheric zonal winds, Nature, 421, 36, 2003. 